\title{
The LS category of the product of lens spaces
}

\author{
ALEXANDER N DRANISHNIKOV
}

\begin{abstract}
We reduce Rudyak's conjecture that a degree-one map between closed manifolds cannot raise the Lusternik-Schnirelmann category to the computation of the category of the product of two lens spaces $L_{p}^{n} \times L_{q}^{n}$ with relatively prime $p$ and $q$. We have computed $\operatorname{cat}\left(L_{p}^{n} \times L_{q}^{n}\right)$ for values $p, q>n / 2$. It turns out that our computation supports the conjecture.

For spin manifolds $M$ we establish a criterion for the equality cat $M=\operatorname{dim} M-1$, which is a K-theoretic refinement of the Katz-Rudyak criterion for cat $M=\operatorname{dim} M$. We apply it to obtain the inequality $\operatorname{cat}\left(L_{p}^{n} \times L_{q}^{n}\right) \leq 2 n-2$ for all odd $n$ and odd relatively prime $p$ and $q$.
\end{abstract}

$55 \mathrm{M} 30 ; 55 \mathrm{~N} 15$

\section{Introduction}

This paper was motivated by the following conjecture of Rudyak:

Conjecture 1.1 [19] A degree-one map between closed manifolds cannot raise the Lusternik-Schnirelmann category.

It is known that degree-one maps $f: M \rightarrow N$ between manifolds tend to have domain more complex than their image. The Lusternik-Schnirelmann category is a numerical invariant that measures the complexity of a space. Thus, Rudyak's conjecture that cat $M \geq$ cat $N$ for a degree-one map $f: M \rightarrow N$ is quite natural. Rudyak (see also the book by Cornea, Lupton, Opera and Tanré [7, page 65]) obtained some partial results supporting the conjecture. In particular, he proved the following:

Theorem 1.2 [19] Let $f: M \rightarrow N$ be a degree- \pm 1 map between closed, stably parallelizable $n$-manifolds, $n \geq 4$, such that 2 cat $N \geq n+4$. Then cat $M \geq$ cat $N$.

In this paper we reduce Rudyak's conjecture to the following question about the LS category of the product of two $n$-dimensional lens spaces $(n=2 k-1)$. 
Problem 1.3 Do there exist $n$ and relatively prime $p$ and $q$ such that

$$
\operatorname{cat}\left(L_{p}^{n} \times L_{q}^{n}\right)>n+1 ?
$$

We show that an affirmative answer to this problem gives a counterexample to Rudyak's conjecture.

This paper is devoted to computation of the category of the product $L_{p}^{n} \times L_{q}^{n}$ of lens spaces for relatively prime $p$ and $q$. Here we use the shorthand notation $L_{p}^{n}=$ $L_{p}^{n}\left(\ell_{1}, \ldots, \ell_{k}\right)$ for a general lens space of dimension $n=2 k-1$, defined for the linear $\mathbb{Z}_{p}$-action on $S^{n} \subset \mathbb{C}^{k}$ determined by the set of natural numbers $\left(\ell_{1}, \ldots, \ell_{k}\right)$ with $\left(p, \ell_{i}\right)=1$ for all $i$.

The obvious inequality cat $X \leq \operatorname{dim} X$ and the cup-length lower bound (see Proposition 2.9) give the estimates

$$
n+1 \leq \operatorname{cat}\left(L_{p}^{n} \times L_{q}^{n}\right) \leq 2 n .
$$

In this paper we prove that, for fixed $n$, the lower bound is almost always sharp.

Theorem 1.4 For every $n=2 k-1$ and primes $p, q \geq k, p \neq q$, for all lens spaces $L_{p}^{n}$ and $L_{q}^{n}$,

$$
\operatorname{cat}\left(L_{p}^{n} \times L_{q}^{n}\right)=n+1 .
$$

This result still leaves some hope to have $\operatorname{cat}\left(L_{p}^{n} \times L_{q}^{n}\right)>n+1$ for small values of $p$ (especially for $p=2$ ) for some lens spaces.

In the second part of the paper we make an improvement of the upper bound in $(*)$. The first improvement comes easily by virtue of the Katz-Rudyak criterion [13]: for a closed $m$-manifold $M$ the inequality cat $(M) \leq m-1$ holds if and only if $M$ is inessential. We recall that Gromov calls a $m$-manifold $M$ inessential if a map $u: M \rightarrow B \pi$ that classifies its universal covering can be deformed to the $(m-1)-$ dimensional skeleton $B \pi^{(m-1)}$. Since for relatively prime $p$ and $q$ the product $L_{p}^{n} \times L_{q}^{n}$ is inessential, we have $\operatorname{cat}\left(L_{p}^{n} \times L_{q}^{n}\right) \leq 2 n-1$. In the paper we improve this inequality to the following:

Theorem 1.5 For all odd $n$ and odd relatively prime $p$ and $q$,

$$
\operatorname{cat}\left(L_{p}^{n} \times L_{q}^{n}\right) \leq 2 n-2 .
$$

For that we study a general question: when is the LS category of a closed spin $m-$ manifold $M$ less than $m-1$ ? We prove in Theorem 6.6 that for a closed $m$-manifold $M$ with $\pi_{2}(M)=0$, the inequality cat $M \leq m-2$ holds if and only if the map $u: M \rightarrow B \pi$ 
can be deformed to the $(m-2)$-dimensional skeleton $B \pi^{(m-2)}$. A deformation of a classifying map of a manifold to the $(m-2)$-skeleton $B \pi^{(m-2)}$ is closely related to Gromov's conjecture on manifolds with positive scalar curvature and it was investigated by Bolotov and Dranishnikov [3]. Combining this with some ideas from [3], we produce a criterion for when a closed spin $m$-manifold $M$ has cat $M \leq m-2$. The criterion involves the vanishing of the integral homology and $k o$-homology fundamental classes of $M$ under a map classifying the universal covering of $M$.

Theorem 1.6 (Criterion) If $M$ is a a closed, spin, inessential $m$-manifold with $\pi_{2}(M)=0$, then

$$
\text { cat } M \leq \operatorname{dim} M-2
$$

if and only if $j_{*} u_{*}\left([M]_{k o}\right)=0$, where $j: B \pi \rightarrow B \pi / B \pi^{(m-2)}$ is the quotient map.

Since a closed orientable manifold $M$ is inessential if and only if $u_{*}([M])=0$ in $H_{*}(B \pi)$ - see Babenko [1] — the Katz-Rudyak criterion for orientable manifolds can be rephrased as follows: cat $M \leq m-1$ if and only if $u_{*}([M])=0$. Thus, our criterion is a further refinement of the Katz-Rudyak criterion.

It turns out that the vanishing of $u_{*}([M])$ in $H_{*}(B \pi)$ makes the primary obstruction to a deformation of $u: M \rightarrow B \pi$ to $B \pi^{(m-2)}$ trivial. It is not difficult to show that the second obstruction lives in the group of coinvariants $\pi_{m}(B \pi, B \pi)_{\pi}$; see [3]. We prove that the group of coinvariants $\pi_{m}\left(B \pi, B \pi^{(m-2)}\right)_{\pi}$ naturally injects into the homotopy group $\pi_{m}\left(B \pi / B \pi^{(m-2)}\right)$. This closes a gap in the computation of the second obstruction in [3]. Based on that injectivity result we use the real connective $\mathrm{K}$-theory to express the second obstruction in terms of the image of the $k o$-fundamental class. The spin condition is needed for the existence of a fundamental class in $k o$-theory. The new upper bound implies that $\operatorname{cat}\left(L_{p}^{3} \times L_{q}^{3}\right)=4$ for all $p$ and $q$. Note that for prime $p$ and $q$ this fact can be also derived from Theorem 1.4.

We complete the paper with a proof of the upper bound formula for the category of a connected sum of two manifolds:

Theorem 1.7

cat $M \# N \leq \max \{$ cat $M$, cat $N\}$.

Since we use this formula in the paper and its original proof in [16] does not cover all cases, we supply an alternative proof.

\section{Acknowledgments}

The author is very grateful to the anonymous referee for many valuable remarks and comments. The author was partially supported by NSF grant DMS-1304627. The author would like to thank the Max-Planck Institut für Mathematik for the hospitality. 


\section{Preliminaries}

\subsection{LS category}

The Lusternik-Schnirelmann category, for a topological space $X$, satisfies cat $X \leq k$ if there is a cover $X=U_{0} \cup \cdots \cup U_{k}$ by $k+1$ open subsets each of which is contractible in $X$. The subsets contractible in $X$ will be called in this note $X$-contractible and The covers of $X$ by subsets contractible in $X$ will be called categorical.

Let $\pi=\pi_{1}(X)$. We recall that the cup product $\alpha \smile \beta$ of twisted cohomology classes $\alpha \in H^{i}(X ; L)$ and $\beta \in H^{j}(X ; M)$ takes values in $H^{i+j}(X ; L \otimes M)$, where $L$ and $M$ are $\pi$-modules and $L \otimes M$ is the tensor product over $\mathbb{Z}$; see Brown [5]. Then the cup-length of $X$, denoted as $\operatorname{cl}(X)$, is defined as the maximal integer $k$ such that $\alpha_{1} \smile \cdots \smile \alpha_{k} \neq 0$ for some $\alpha_{i} \in H^{n_{i}}\left(X ; L_{i}\right)$ with $n_{i}>0$. The following inequalities give estimates on the LS category:

Theorem 2.1 [7] $\quad \operatorname{cl}(X) \leq$ cat $X \leq \operatorname{dim} X$.

\subsection{Ganea-Schwarz approach to the LS category}

Given two maps $f_{1}: X_{1} \rightarrow Y$ and $f_{2}: X_{2} \rightarrow Y$, we set

$$
Z=\left\{\left(x_{1}, x_{2}, t\right) \in X_{1} * X_{2} \mid f_{1}\left(x_{1}\right)=f_{2}\left(x_{2}\right)\right\}
$$

and define the fiberwise join, or join over $Y$, of $f_{1}$ and $f_{2}$ as the map

$$
f_{1} *_{Y} f_{2}: Z \rightarrow Y, \quad\left(f_{1} *_{Y} f_{2}\right)\left(x_{1}, x_{2}, t\right)=f_{1}\left(x_{1}\right)=f_{2}\left(x_{2}\right) .
$$

Let $p_{0}^{X}: P X \rightarrow X$ be the Serre path fibration. This means that $P X$ is the space of paths on $X$ that start at the base point $x_{0} \in X$, and $p_{0}^{X}(\alpha)=\alpha(1)$ for $\alpha \in P X$. We denote by $p_{n}^{X}: G_{n}(X) \rightarrow X$ the iterated fiberwise join of $n+1$ copies of $p_{0}^{X}$. Thus, the fiber $F_{n}=\left(p_{n}^{X}\right)^{-1}\left(x_{0}\right)$ of the fibration $p_{n}^{X}$ is the join product $\Omega X * \cdots * \Omega X$ of $n+1$ copies of the loop space $\Omega X$ on $X$. So, $F_{n}$ is $(n-1)$-connected. It is known that $G_{n}(X)$ is homotopy equivalent to the mapping cone of the inclusion of the fiber $F_{n-1} \rightarrow G_{n-1}(X)$.

When $X=K(\pi, 1)$, the loop space $\Omega X$ is naturally homotopy equivalent to $\pi$ and the space $G_{n}(\pi)=G_{n}(K(\pi, 1))$ has the homotopy type of a $n$-dimensional complex. The proof of the following theorem can be found in [7]:

Theorem 2.2 (Ganea, Schwarz) For a $C W$ space $X$, cat $(X) \leq n$ if and only if there exists a section of $p_{n}^{X}: G_{n}(X) \rightarrow X$. 
This theorem can be extended to maps:

Theorem 2.3 For a map $f: Y \rightarrow X$ to a $C W$ space $X$, cat $(f) \leq n$ if and only if there exists a lift of $f$ with respect to $p_{n}^{X}: G_{n}(X) \rightarrow X$.

We recall that the LS category of a map $f: Y \rightarrow X$ is the least integer $k$ for which $Y$ can be covered by $k+1$ open sets $U_{0}, \ldots, U_{k}$ such that the restrictions $\left.f\right|_{U_{i}}$ are null-homotopic for all $i$.

We use the notation $\pi_{*}(f)=\pi_{*}\left(M_{f}, X\right)$, where $M_{f}$ is the mapping cylinder of $f: X \rightarrow Y$. Then $\pi_{i}(f)=0$ for $i \leq n$ amounts to saying that $f$ induces isomorphisms $f_{*}: \pi_{i}(X) \rightarrow \pi_{i}(Y)$ for $i<n$ and an epimorphism in dimension $n$.

Proposition 2.4 [8] Let $f_{j}: X_{j} \rightarrow Y_{j}, 3 \leq j \leq s$ be a family of maps of CW spaces such that $\pi_{i}\left(f_{j}\right)=0$ for $i \leq n_{j}$. Then the joins satisfy

$$
\pi_{k}\left(f_{1} * f_{2} * \cdots * f_{s}\right)=0
$$

for $k \leq \min \left\{n_{j}\right\}+s-1$.

\subsection{The Berstein-Schwarz class}

Let $\pi$ be a discrete group and $A$ be a $\pi$-module. By $H^{*}(\pi, A)$ we denote the cohomology of the group $\pi$ with coefficients in $A$ and by $H^{*}(X ; A)$ we denote the cohomology of a space $X$ with the twisted coefficients defined by $A$. The BersteinSchwarz class of a group $\pi$ is a certain cohomology class $\beta_{\pi} \in H^{1}(\pi, I(\pi))$, where $I(\pi)$ is the augmentation ideal of the group ring $\mathbb{Z} \pi$; see Berstein [2] and Schwarz [22]. It is defined as the first obstruction to a lift of $B \pi=K(\pi, 1)$ to the universal covering $E \pi$. The class $\beta_{\pi}$ is defined by a cocycle $\beta: E \pi^{(1)} \rightarrow I(\pi)$. We note that the 1-skeleton of $E \pi$ can be identified with the Cayley graph of $\pi$. For a fixed set $S$ of generators of $\pi$, the Cayley graph $C=C(\pi, S)$ has $V=\pi$ as the set of vertices and $E=\{[\gamma, \gamma s] \mid \gamma \in \pi, s \in S\}$ as the set of edges.

Note that the 1-skeleton of $B \pi$ can be identified with the wedge of circles labeled by $S$. Then the $1-$ skeleton $E \pi^{(1)}$ of the universal covering equals the Cayley graph $C=C(\pi, S)$. In that case the cocycle $\beta$ takes every edge $[a, b] \subset C$ to $b-a \in I(\pi)$.

Here is a more algebraic definition of $\beta_{\pi}$. Consider the cohomology long exact sequence generated by the short exact sequence of coefficients

$$
0 \longrightarrow I(\pi) \longrightarrow \mathbb{Z} \pi \stackrel{\epsilon}{\longrightarrow} \mathbb{Z} \longrightarrow 0,
$$


where $\epsilon$ is the augmentation homomorphism. Then $\beta_{\pi}=\delta(1)$ equals the image of the generator $1 \in H^{0}(\pi ; \mathbb{Z})=\mathbb{Z}$ under the connecting homomorphism

$$
\delta: H^{0}(\pi ; \mathbb{Z}) \rightarrow H^{1}(\pi ; I(\pi))
$$

It follows from the definition of the connecting homomorphism $\delta$ (snake lemma) that $\delta(1)$ is defined by the above cocycle $\beta$.

Theorem 2.5 (Universality [9; 22]) For any cohomology class $\alpha \in H^{k}(\pi, L)$ there is a homomorphism of $\pi$-modules $I(\pi)^{k} \rightarrow L$ such that the induced homomorphism for cohomology takes $\left(\beta_{\pi}\right)^{k} \in H^{k}\left(\pi ; I(\pi)^{k}\right)$ to $\alpha$, where $I(\pi)^{k}=I(\pi) \otimes \cdots \otimes I(\pi)$ and $\left(\beta_{\pi}\right)^{k}=\beta_{\pi} \smile \cdots \smile \beta_{\pi}$.

Corollary 2.6 [22] The class $\left(\beta_{\pi}\right)^{n+1}$ is the primary obstruction to a section of $p_{n}^{B \pi}: G_{n}(\pi) \rightarrow B \pi$.

Corollary 2.7 For any group $\pi$, its cohomological dimension can be expressed as

$$
\operatorname{cd}(\pi)=\max \left\{n \mid\left(\beta_{\pi}\right)^{n} \neq 0\right\} .
$$

Corollary 2.8

$$
\operatorname{cl}\left(L_{p}^{n}\right)=n
$$

Proof For any lens space $L_{p}^{n}$ the inclusion $L_{p}^{n} \rightarrow B \mathbb{Z}_{p}$ to the classifying space as the $n$-skeleton takes $\left(\beta_{\mathbb{Z}_{p}}\right)^{n}$ to a nonzero element $\beta^{n}$. Since $\operatorname{cd}\left(\mathbb{Z}_{p}\right)=\infty$, we obtain $\left(\beta_{\mathbb{Z}_{p}}\right)^{n} \neq 0$. Since the restriction to the $n$-skeleton is injective on $n$-dimensional cohomology groups, the result follows.

Proposition 2.9

$$
\operatorname{cl}\left(L_{p}^{n} \times L_{q}^{n}\right) \geq n+1 .
$$

Proof Let $\alpha \in H^{n}\left(L_{q}^{n}\right)=\mathbb{Z}$ be a generator. Then, in view of the Kunneth formula for local coefficients [4], the cross product

$$
\beta^{n} \times \alpha \in H^{2 n+1}\left(L_{p}^{n} \times L_{q}^{n} ; I\left(\mathbb{Z}_{p}\right)^{n}\right)
$$

is nontrivial for the above $\beta \in H^{1}\left(L_{p}^{n} ; I\left(\mathbb{Z}_{p}\right)\right)$. 


\section{Some examples of degree-one maps}

Let $M$ be an oriented manifold and $k \in \mathbb{Z} \backslash\{0\}$; by $k M$ we denote the connected sum $M \# \cdots \# M$ of $|k|$ copies of $M$, taken with the opposite orientation if $k$ is negative. For an odd $n$ and natural $p>1$ we denote by $L_{p}^{n}$ a lens space, ie the orbit space $S^{n} / \mathbb{Z}_{p}$ for a free linear action of $\mathbb{Z}_{p}=\mathbb{Z} / p \mathbb{Z}$ on the sphere $S^{n}$.

Theorem 3.1 For $m, n \in 2 \mathbb{N}+1$ and any relatively prime numbers $p$ and $q$ there are $k, l \in \mathbb{Z}$ such that the manifold

$$
M=k\left(L_{p}^{m} \times S^{n}\right) \# l\left(S^{m} \times L_{q}^{n}\right)
$$

admits a degree-one map $\phi: M \rightarrow N$ onto $N=L_{p}^{m} \times L_{q}^{n}$.

Proof Take $k$ and $l$ such that $l p+k q=1$. Let $f: S^{m} \rightarrow L_{p}^{m}$ and $g: S^{n} \rightarrow L_{q}^{n}$ be the projections to the orbit space for the $\mathbb{Z}_{p}$ and $\mathbb{Z}_{q}$ free actions, respectively. We may assume that the above connected sum is obtained by taking the wedge of $|k|+|l|-1$ spheres of dimension $m+n-1$ embedded in one of the summands and gluing all other summands along those spheres. Consider the quotient map

$$
\psi: k\left(L_{p}^{m} \times S^{n}\right) \# l\left(S^{m} \times L_{q}^{n}\right) \rightarrow \bigvee_{k}\left(L_{p}^{m} \times S^{n}\right) \vee \bigvee_{l}\left(S^{m} \times L_{q}^{n}\right)
$$

that collapses the wedge of those $(m+n-1)-$ spheres to a point. Let the map

$$
\phi: \bigvee_{k}\left(L_{p}^{m} \times S^{n}\right) \vee \bigvee_{l}\left(S^{m} \times L_{q}^{n}\right) \rightarrow L_{p}^{m} \times L_{q}^{n}
$$

be defined as the union

$$
\phi=\bigcup_{k}(1 \times g) \cup \bigcup_{l}(f \times 1) .
$$

Note that the degree of $f \times 1$ is $p$, the degree of $1 \times g$ is $q$ and the degree of $\phi \circ \psi$ is $l p+k q=1$.

Proposition 3.2 For $m \leq n, \operatorname{cat}\left(k\left(L_{p}^{m} \times S^{n}\right) \# l\left(S^{m} \times L_{q}^{n}\right)\right)=n+1$.

Proof It follows from the cup-length estimate that cat $\left(S^{m} \times L_{q}^{n}\right) \geq n+1$ and, generally, $\operatorname{cat}\left(k\left(L_{p}^{m} \times S^{n}\right) \# l\left(S^{m} \times L_{q}^{n}\right)\right) \geq n+1$ when $l \neq 0$. By the product formula, $\operatorname{cat}\left(S^{m} \times L_{r}^{n}\right) \leq n+1$. Thus, $\operatorname{cat}\left(S^{m} \times L_{r}^{n}\right)=n+1$. Then, by the sum formula [16] (see Theorem 7.1),

$$
\operatorname{cat}\left(k\left(L_{p}^{m} \times S^{n}\right) \# l\left(S^{m} \times L_{q}^{n}\right)\right) \leq n+1 .
$$


Now one can see the connection between Rudyak's conjecture and Problem 1.3. If there exist relatively prime $p$ and $q$ and odd $n$ such that $\operatorname{cat}\left(L_{p}^{n} \times L_{q}^{n}\right)>n+1$, then the map of Theorem 3.1 will be a counter-example to Rudyak's conjecture.

Remark In Theorem 3.1 one can use fake lens spaces. Since every fake lens space is homotopy equivalent to a lens space [23] and the LS category is a homotopy invariant, it suffices to consider only the classical lens spaces.

\section{On the category of the product of lens spaces}

Let $\bar{\ell}=\left(\ell_{1}, \ldots, \ell_{k}\right)$ be a set of $\bmod p$ integers relatively prime to $p$. The lens space $L_{p}^{2 k-1}(\bar{\ell})$ is the orbit space of the action of $\mathbb{Z}_{p}=\langle t\rangle$ on the unit sphere $S^{2 k-1} \subset \mathbb{C}^{k}$ defined by the formula

$$
t\left(z_{1}, \ldots, z_{k}\right)=\left(e^{2 \pi i \ell_{1} / p} z_{1}, \ldots, e^{2 p i i \ell_{k} / p}\right) .
$$

We note that for all $k$ the lens spaces $L_{p}^{2 k-1}(\bar{\ell})$ have a natural $\mathrm{CW}$ complex structure with one cell in each dimension up to $2 k-1$ such that $L_{p}^{2 k-1}(\bar{\ell})$ is the $(2 k-1)-$ skeleton of $L_{p}^{2 k+1}\left(\bar{\ell}, \ell_{k+1}\right)$. If $\alpha: \mathbb{Z}_{p} \times S^{2 k-1} \rightarrow S^{2 k-1}$ is a free action which is not necessarily linear, its orbit space is called a fake lens space and is denoted by $L_{p}^{2 k-1}(\alpha)$.

We recall that a closed, oriented $n$-manifold $M$ is called inessential-see Gromov [12] - if a map $u: M \rightarrow B \pi=K(\pi, 1)$ that classifies its universal cover can be deformed to the $(n-1)$-dimensional skeleton $B \pi^{(n-1)}$. It is known that a closed, oriented $n$-manifold $M$ is essential if and only if $u_{*}([M]) \neq 0$, where $[M] \in H_{n}(M ; \mathbb{Z})$ denotes the fundamental class $[1 ; 3]$.

We note that cat $M=\operatorname{dim} M$ if and only if $M$ is essential [13]. Clearly, every lens space $L_{p}^{n}$ is essential. In particular, cat $L_{p}^{n}=n$. Since $\mathbb{Z}_{p} \otimes \mathbb{Z}_{q}=0$ for relatively prime $p$ and $q$, the product $L_{p}^{m} \times L_{q}^{n}$ is inessential. Hence, $\operatorname{cat}\left(L_{p}^{m} \times L_{q}^{n}\right) \leq m+n-1$ for all $p$ and $q$.

\subsection{Stably parallelizable lens spaces}

First we do our computation for stably parallelizable lens spaces.

Proposition 4.1 For lens spaces $L_{p}^{m}$ and $L_{q}^{n}$ with $m \leq n$ and $(p, q)=1$ which are homotopy equivalent to stably parallelizable manifolds,

$$
\operatorname{cat}\left(L_{p}^{m} \times L_{q}^{n}\right)=n+1 .
$$




\section{Proof Let}

$$
\phi: M=k\left(L_{p}^{m} \times S^{n}\right) \# l\left(S^{m} \times L_{q}^{n}\right) \rightarrow N=L_{p}^{m} \times L_{q}^{n}
$$

be the map of degree one from Theorem 3.1. Suppose that $L_{p}^{m}$ and $L_{q}^{n}$ are homotopy equivalent to stably parallelizable manifolds $N_{p}^{m}$ and $N_{q}^{n}$, respectively. Then there are homotopy equivalences $h: M^{\prime}=k\left(N_{p}^{m} \times S^{n}\right) \# l\left(S^{m} \times N_{q}^{n}\right) \rightarrow M$ and $h^{\prime}: N=L_{p}^{m} \times L_{q}^{n} \rightarrow N^{\prime}=N_{p}^{m} \times N_{q}^{n}$. Since a connected sum and the product of stably parallelizable manifolds are stably parallelizable (see for example [14]), the manifolds $M^{\prime}$ and $N^{\prime}$ are stably parallelizable. Assume that $\operatorname{cat}\left(L_{p}^{m} \times L_{q}^{n}\right) \geq n+2$. Then

$$
2 \text { cat } N^{\prime}=2 \operatorname{cat}\left(L_{p}^{m} \times L_{q}^{n}\right)=2(n+2) \geq m+n+4=\operatorname{dim}\left(L_{p}^{m} \times L_{q}^{n}\right)+4 .
$$

By Theorem 1.2 applied to the map $h^{\prime} \circ \phi \circ h: M^{\prime} \rightarrow N^{\prime}$ from Theorem 3.1, we obtain a contradiction:

$$
n+2=\text { cat } N=\text { cat } N^{\prime} \leq \text { cat } M^{\prime}=\text { cat } M=n+1 .
$$

Since all orientable 3-manifolds are stably parallelizable, we obtain:

Corollary 4.2 For relatively prime $p$ and $q$,

$$
\operatorname{cat}\left(L_{p}^{3} \times L_{q}^{3}\right)=4
$$

There is a characterization of stable parallelizability of lens spaces [10]: the lens space $L_{p}^{2 k-1}\left(\ell_{1}, \ldots, \ell_{k}\right)$ is stably parallelizable if and only if $p \geq k$ and $\ell_{1}^{2 j}+\cdots+\ell_{k}^{2 j}=$ $0 \bmod p$ for $j=1,2, \ldots,\left[\frac{1}{2}(k-1)\right]$. We recall that two lens spaces $L_{p}^{2 k-1}\left(\ell_{1}, \ldots, \ell_{k}\right)$ and $L_{p}^{2 k-1}\left(\ell_{1}^{\prime}, \ldots, \ell_{k}^{\prime}\right)$ are homotopy equivalent [17] if and only if the $\bmod p$ equation

$$
\ell_{1} \ell_{2} \cdots \ell_{k}= \pm a^{k} \ell_{1}^{\prime} \ell_{2}^{\prime} \cdots \ell_{k}^{\prime}
$$

has a solution $a \in \mathbb{Z}_{p}$. These conditions imply that a lens space is rarely homotopy equivalent to a stably parallelizable one. Nevertheless, Ewing, Moolgavkar, Smith and Stong [10] showed that, for each $n=2 k-1$, for infinitely many primes $p$ there are stably parallelizable lens spaces $L_{p}^{n}$. Clearly, there are more chances for the existence of stably parallelizable fake lens spaces with given $n$ and $p$. Thus, Kwak [15] proved that for every odd $n=2 k-1$ and $p \geq k$ there is a fake $n$-dimensional stably parallelizable lens space. Since every fake lens space is homotopy equivalent to a lens space - see Wall [23] - we obtain that for every $n=2 k-1$ and $p \geq k$ there is a lens space $L_{p}^{n}$ homotopy equivalent to a stably parallelizable manifold. 


\subsection{Category of classifying maps}

We recall that any map $u: X \rightarrow B \pi=K(\pi, 1)$ of a CW complex $X$ that induces an isomorphism of the fundamental group classifies the universal covering $\tilde{X}$, ie $\tilde{X}$ is obtained as the pull-back of the universal covering $E \pi$ of $B \pi$ by means of $u$. We call such a map a classifying map of $X$.

Proposition 4.3 Let $u: X \rightarrow B \pi$ be a map classifying the universal covering of a $C W$ complex $X$. Then the following are equivalent:

(1) $\operatorname{cat}(u) \leq k$.

(2) $u$ admits a lift $u^{\prime}: X \rightarrow G_{k}(\pi)$ of $u$ with respect to $p_{n}^{\pi}: G_{k}(\pi) \rightarrow B \pi$.

(3) $u$ is homotopic to a map $f: X \rightarrow B \pi$ with $f(X) \subset B^{(k)}$.

Proof $(1) \Longrightarrow(2)$ is a part of Theorem 2.3.

(2) $\Longrightarrow$ (3) Since $G_{k}(\pi)$ has the homotopy type of a $k$-dimensional complex, the map $p_{k}^{\pi}$ can be deformed to a map $p^{\prime}$ with the image in $B \pi^{(k)}$. Then we can take $f=p^{\prime} \circ u^{\prime}$.

(3) $\Longrightarrow$ (1) For a map $f: X \rightarrow B \pi$ with $f(X) \subset B^{(k)}$ homotopic to $u$ we obtain $\operatorname{cat}(u)=\operatorname{cat}(f) \leq$ cat $B \pi^{(k)} \leq k$.

Theorem 4.4 Let $X$ be an $n$-dimensional $C W$ complex with a classifying map $u: X \rightarrow B \pi$ having cat $u \leq k$ and with $(n-k)$-connected universal covering $\tilde{X}$. Then cat $X \leq k$.

Proof Note that the map $p_{k}^{X}$ factors through the pull-back, $p_{k}^{X}=p^{\prime} \circ q$ :

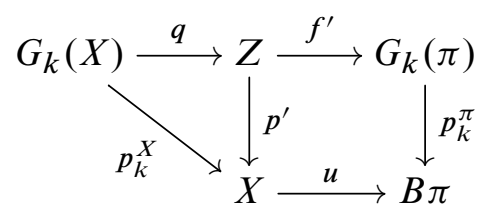

The condition cat $u \leq k$ implies that $u$ has a lift $u^{\prime}: X \rightarrow G_{k}(\pi), u=p_{k}^{\pi} u^{\prime}$. Hence, $p^{\prime}$ admits a section $s: X \rightarrow Z$. Since $X$ is $n$-dimensional, to show that $s$ has a lift with respect to $q$ it suffices to prove that the homotopy fiber $F$ of the map $q$ is $(n-1)$-connected. Since $\pi_{i}(X)=0$ for $1<i \leq n-k, B \pi$ is aspherical and $u$ induces an isomorphism of the fundamental groups, we obtain $\pi_{i}(u)=0$ for $i \leq n-k+1$. Hence, $\pi_{i}(\Omega u)=0$ for $i \leq n-k$. Then, by Proposition 2.4, $\pi_{i}\left(*_{k+1} \Omega u\right)=0$ for 
$i \leq(n-k)+(k+1)-1=n$. The commutative diagram generated by $q$ and the fibrations $p_{X}^{k}$ and $p^{\prime}$,

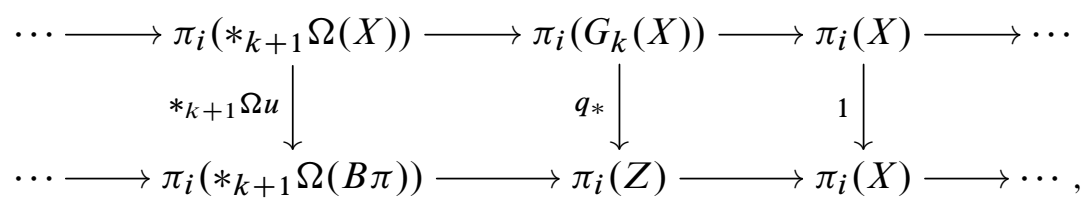

and the five lemma imply that $\pi_{i}(q)=0$ for $i \leq n$. Hence, $\pi_{i}(F)=0$ for $i \leq n-1$. Thus, $s$ admits a homotopy lift. Therefore, $p_{k}^{X}$ has a homotopy section and, hence, it admits a section. Therefore, by Theorem 2.2, cat $X \leq k$.

\subsection{The main computation}

Proposition 4.5 For any two lens spaces $L_{p}^{n}(\bar{\ell})$ and $L_{p}^{n}(\bar{\mu})$, there is a map

$$
f: L_{p}^{n}(\bar{\ell}) \rightarrow L_{p}^{n}(\bar{\mu})
$$

that induces an isomorphism of the fundamental groups.

Proof Let $q_{1}: S^{n} \rightarrow L_{p}^{n}(\bar{\ell})$ and $q_{2}: S^{n} \rightarrow L_{p}^{n}(\bar{\mu})$ be the projections onto the orbit spaces of the corresponding $\mathbb{Z}_{p}$-actions. We note that $L_{p}^{n}(\bar{\mu})$ is the $n$-skeleton in $L_{p}^{n+2}(\bar{\mu}, 1)$. Let $\bar{q}_{2}: S^{n+2} \rightarrow L_{p}^{n}(\mu, 1)$ be the corresponding projection:

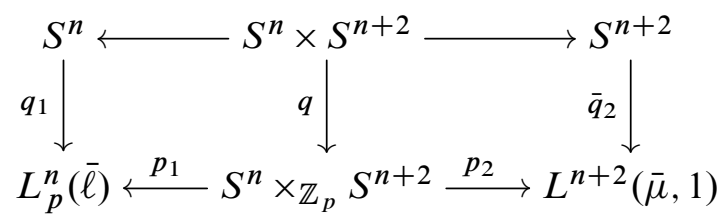

Since in the Borel construction for the diagonal $\mathbb{Z}_{p}$ action on $S^{n} \times S^{n+2}$ the projection $p_{1}$ is $(n+1)$-connected, it admits a section $s: L_{p}^{n}(\bar{\ell}) \rightarrow S^{n} \times \mathbb{Z}_{p} S^{n+2}$. Then $f$ is a cellular approximation of $p_{2} \circ s$.

Theorem 4.6 For every odd $n=2 k-1$ and distinct primes $p, q \geq k$,

$$
\operatorname{cat}\left(L_{p}^{2 k-1} \times L_{q}^{2 k-1}\right)=n+1 .
$$

Proof Let $L_{p}^{n}=L_{p}^{n}(\bar{\ell})$ and $L_{q}^{n}\left(\bar{\ell}^{\prime}\right)$ for $\bar{\ell}=\left(\ell_{1}, \ldots, \ell_{k}\right)$ and $\bar{\ell}^{\prime}=\left(\ell_{1}^{\prime}, \ldots, \ell_{k}^{\prime}\right)$. By Kwak [15, Theorem 3.1] there are stably parallelizable fake lens spaces $L_{p}^{n}(\alpha)$ and $L_{q}^{n}\left(\alpha^{\prime}\right)$. By Wall's theorem they are homotopy equivalent to lens spaces $L_{p}^{n}(\bar{\mu})$ and $L_{q}^{n}\left(\bar{\mu}^{\prime}\right)$ for some $\bar{\mu}$ and $\bar{\mu}^{\prime}$. By Proposition 4.1, $\operatorname{cat}\left(L_{p}^{n}(\bar{\mu}) \times L_{q}^{n}\left(\bar{\mu}^{\prime}\right)\right)=n+1$. 
By Proposition 4.3, there is a classifying map $u$ : $L_{p}^{n}(\bar{\mu}) \times L_{q}^{n}\left(\bar{\mu}^{\prime}\right) \rightarrow B \mathbb{Z}_{p q}^{(n+1)}$. By Proposition 4.5 there are maps $f_{p}: L_{p}^{n} \rightarrow L_{p}^{n}(\bar{\mu})$ and $f_{q}: L_{q}^{n} \rightarrow L_{q}^{n}\left(\bar{\mu}^{\prime}\right)$ that induce an isomorphism of the fundamental groups. Therefore,

$$
u^{\prime}=u \circ\left(f_{p} \times f_{q}\right): L_{p}^{n} \times L_{q}^{n} \rightarrow B \mathbb{Z}_{p q}^{(n+1)}
$$

is a classifying map for $L_{p}^{n} \times L_{q}^{n}$. Hence, $\operatorname{cat}\left(u^{\prime}\right) \leq n+1$. Since the universal covering of the space $L_{p}^{n} \times L_{q}^{n}$ is $(n-1)$-connected, by Theorem 4.4 we obtain cat $\left(L_{p}^{n} \times L_{q}^{n}\right) \leq n+1$. By Proposition 2.9, $\operatorname{cat}\left(L_{p}^{n} \times L_{q}^{n}\right)=n+1$.

Remark When $p$ and $q$ are relatively prime but not necessarily prime we can prove the equality $\operatorname{cat}\left(L_{p}^{n} \times L_{q}^{n}\right)=n+1$ with a stronger restriction $p, q \geq n+3$. We do not present the proof, since it is more technical. It consists of computation of obstructions for deforming a classifying map $u: L_{p}^{n} \times L_{q}^{n} \rightarrow B \mathbb{Z}_{p q}$ to the $(n+1)-$ skeleton. Vanishing of the first obstruction happens without any restriction on $p$ and $q$. Since it is a curious fact on its own it is presented in the next section. The higher obstructions vanish due to the fact that cohomology groups of $\mathbb{Z}_{p q}$ are $p q$-torsions and a theorem of Serre [20] that states that the group $\pi_{n+k}\left(S^{n}\right)$ has zero $r$-torsion component for $k<2 r-4$.

We note that Theorem 4.6 can be stated for all lens spaces $L_{p}^{n}$ with values of $n$ and $p$ for which there exists a stably parallelizable fake lens space $L_{p}^{n}(\alpha)$.

Problem 4.7 For which values of $n$ and $p$ is there a stably parallelizable fake lens space $L_{k}^{n}(\alpha)$ ?

This does not seem to happen very often when $p=2$. At least, a real $(2 k-1)-$ dimensional projective space is stably parallelizable if and only if $k=1,2$ or 4 .

\section{The Berstein-Schwarz class for the product of finite cyclic groups}

Let $u: L_{p}^{n} \times L_{q}^{n} \rightarrow B \mathbb{Z}_{p q}$ be a classifying map. By Theorem 4.4 and the fact that $\operatorname{cat}\left(L_{p}^{n} \times L_{q}^{n}\right) \geq n+1$, the condition $\operatorname{cat}(u) \leq n+1$ is equivalent to the equality $\operatorname{cat}\left(L_{p}^{n} \times L_{q}^{n}\right)=n+1$. By Proposition 4.3 the inequality cat $(u) \leq n+1$ is equivalent to the existing of a lift $u^{\prime}$ of $u$ with respect to $p_{n}: G_{n+1}\left(\mathbb{Z}_{p q}\right) \rightarrow B \mathbb{Z}_{p q}$. In view of Corollary 2.6 the primary obstruction to such a lift is $u^{*}\left(\beta^{n+2}\right)$, where $\beta$ is the Berstein-Schwarz class of $\mathbb{Z}_{p q}$. We prove that this obstruction is always zero and even more: 
Theorem 5.1 For all $n$ and all relatively prime $p$ and $q$,

$$
u^{*}\left(\beta^{n+1}\right)=0 .
$$

Remark One can show that for sufficiently large $p$ and $q$ the higher obstructions are trivial as well, since the homotopy groups of the fiber of $p_{n}^{\pi}$ do not contain $r$-torsions for large $r$. This would give a result similar to Theorem 4.6, which does not cover small values of $p$.

We denote by $\mathbb{Z}(m)$ the group ring $\mathbb{Z} \mathbb{Z}_{m}$ of $\mathbb{Z}_{m}, I(m)$ its augmentation ideal, $\epsilon_{m}: \mathbb{Z}(m) \rightarrow \mathbb{Z}$ its augmentation, and $\beta_{m}$ its Berstein-Schwarz class. Let $t_{m}=$ $\sum_{g \in \mathbb{Z}_{m}} g \in \mathbb{Z}(m)$. We use the same notation $t_{m}$ for a constant map $t_{m}: \mathbb{Z}_{m} \rightarrow \mathbb{Z}(m)$ with the value $t_{m}$. We note that the group of invariants of $\mathbb{Z}(m)$ is $\mathbb{Z}$ generated by $t_{m}$. Thus, $H^{0}\left(\mathbb{Z}_{m} ; \mathbb{Z}(m)\right)=\mathbb{Z}$.

Proposition 5.2 Let $\beta_{p}$ denote the Berstein-Schwarz class for the group $\mathbb{Z}_{p}=\mathbb{Z} / p \mathbb{Z}$. Then $\beta_{p}$ has order $p$ and hence is $q$-divisible for any $q$ relatively prime to $p$.

Proof Let $t \in \mathbb{Z}_{p}$ be a generator. We note that

$$
H^{0}\left(\mathbb{Z}_{p} ; \mathbb{Z}_{p}\right)=\left(\mathbb{Z}_{\mathbb{Z}_{p}}\right)^{\mathbb{Z}_{p}}=\mathbb{Z}\left\langle 1+t+\cdots+t^{p-1}\right\rangle
$$

is the group of invariants, which is isomorphic to the subgroup of $\mathbb{Z} \mathbb{Z}_{p}$ generated by $1+t+\cdots+t^{p-1}$. Then the augmentation homomorphism $\epsilon: \mathbb{Z} \mathbb{Z}_{p} \rightarrow \mathbb{Z}$ induces a homomorphism $\epsilon_{*}: H^{0}\left(\mathbb{Z}_{p} ; \mathbb{Z} \mathbb{Z}_{p}\right) \rightarrow H^{0}\left(\mathbb{Z}_{p} ; \mathbb{Z}\right)=\mathbb{Z}$ that takes the generator $1+t+\cdots+t^{p-1}$ to $p$. Thus, $p \beta_{p}=p \delta(1)=\delta(p)=0$ by exactness of the cohomology long exact sequence associated with the coefficient sequence $0 \rightarrow I(p) \rightarrow \mathbb{Z}(p) \rightarrow \mathbb{Z} \rightarrow 0$.

Note that $\beta_{p}$ generates a subgroup $G$ of order $p$ in $H^{1}(\pi ; I(\mathbb{Z} \pi))$. Therefore it is $q$-divisible for $q$ with $(p, q)=1$.

We recall that the cross product

$$
H^{i}(X ; M) \times H^{j}\left(X^{\prime} ; M^{\prime}\right) \rightarrow H^{i+j}\left(X \times X^{\prime} ; M \otimes_{\mathbb{Z}} M^{\prime}\right)
$$

is defined for any $\pi_{1}(X)$-module $M$ and $\pi_{1}\left(X^{\prime}\right)$-module $M^{\prime}$. Also we note that

$$
H^{i}\left(X ; M \oplus M^{\prime}\right)=H^{i}(X ; M) \oplus H^{i}\left(X ; M^{\prime}\right) .
$$

Proposition 5.3 For relatively prime $p$ and $q$ there are $k, l \in \mathbb{Z}$ such that the BersteinSchwarz class $\beta_{p q}$ is the image of the class

$$
\left(\beta_{p} \times l, k \times \beta_{q}\right) \in H^{1}\left(\mathbb{Z}_{p q} ; I(p) \otimes \mathbb{Z}(q)\right) \oplus H^{1}\left(\mathbb{Z}_{p q} ; \mathbb{Z}(p) \otimes I(q)\right)
$$


under the coefficient homomorphism

$$
\phi: I(p) \otimes \mathbb{Z}(q) \oplus \mathbb{Z}(p) \otimes I(q) \rightarrow I(p q) \subset \mathbb{Z}(p q)=\mathbb{Z}(p) \otimes \mathbb{Z}(q)
$$

defined by the inclusions of the direct summands into $\mathbb{Z}(p) \otimes \mathbb{Z}(q)$ and the summation.

Proof Let $k$ and $l$ be such that $k p+l q=1$.

The addition in $\mathbb{Z}(p q)$ defines the commutative diagram

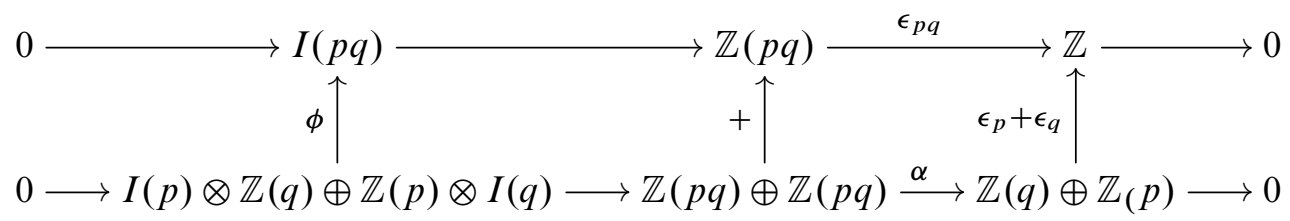

which defines a commutative square for cohomology:

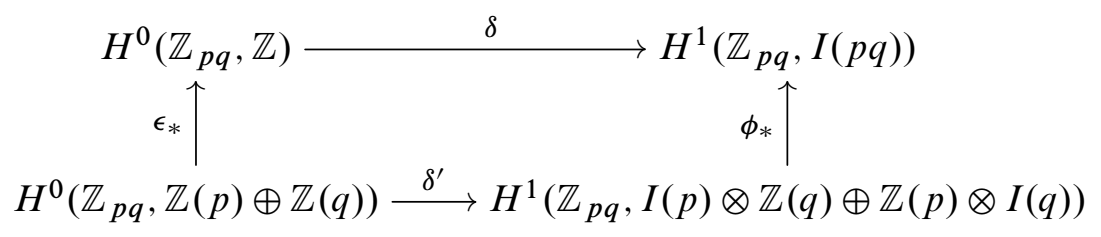

The homomorphism $\theta: \mathbb{Z}(p q) \rightarrow \mathbb{Z}(p) \oplus \mathbb{Z}(q)$ defined on the basis as $\theta(a \times b)=$ $\left(l t_{q}, k t_{p}\right)$ is a cochain since it is $\mathbb{Z}_{p q}$-equivariant. It is a cocycle, since it is constant. Note that $\left(\epsilon_{p}+\epsilon_{q}\right) \circ \theta(a \times b)=k p+l q=1$ for any $a \in \mathbb{Z}_{p}$ and $b \in \mathbb{Z}_{q}$. This means that the cohomology class $[\theta]$ is taken by $\epsilon_{*}$ to a generator $1 \in H^{0}\left(\mathbb{Z}_{p q} ; \mathbb{Z}\right)$. Then $\beta_{p q}=\delta(1)=\phi \delta^{\prime}([\theta])$.

Consider a $\mathbb{Z}(p q)$-homomorphism $\bar{\theta}: \mathbb{Z}(p) \times \mathbb{Z}(q) \rightarrow \mathbb{Z}(p q) \oplus \mathbb{Z}(p q)$ defined by the formula $\bar{\theta}(a \times b)=\left(a \times l t_{q}, k t_{p} \times b\right)$. Since $\alpha(\bar{\theta})=\theta$, by the snake lemma $\delta^{\prime}([\theta])$ is defined by the 1-cocycle $\delta(\bar{\theta}): C_{1} \rightarrow I(p) \otimes \mathbb{Z}(q) \oplus \mathbb{Z}(p) \otimes I(q)$. Note that the cellular 1-dimensional chain group $C_{1}$ is defined via the Cayley graph $C$ of $\mathbb{Z}_{p q}$.

Note that the Cayley graph $C\left(\pi \times \pi^{\prime}, S \times e^{\prime} \cup e \times S^{\prime}\right)$ of the product $\pi \times \pi^{\prime}$ of two groups with generating sets $S$ and $S^{\prime}$ and units $e \in \pi$ and $e^{\prime} \in \pi^{\prime}$ equals the 1-skeleton of the product of the Cayley graphs $C(\pi, S) \times C\left(\pi^{\prime}, S^{\prime}\right)$. Thus, $C=\left(C^{p} \times \mathbb{Z}_{q}\right) \cup\left(\mathbb{Z}_{p} \times C^{q}\right)$, where $C^{p}$ and $C^{q}$ are the Cayley graphs (cycles) for $\mathbb{Z}_{p}$ and $\mathbb{Z}_{q}$, respectively. Note that

$$
\begin{aligned}
\delta(\bar{\theta})\left(\left[a_{1}, a_{2}\right] \times b\right) & =\bar{\theta}\left(\left(a_{2}-a_{1}\right) \times b\right)=\bar{\theta}\left(a_{2} \times b\right)-\bar{\theta}\left(a_{1} \times b\right) \\
& =\left(a_{2} \times l t_{q}, k t_{p} \times b\right)-\left(a_{1} \times l t_{q}, k t_{p} \times b\right)=\left(\left(a_{2}-a_{1}\right) \times l t_{q}, 0\right) \\
& =\left(\beta_{p} \times l t_{q}\right)\left(\left[a_{1}, a_{2}\right] \times b\right)=\left(\beta_{p} \times l t_{q}, k t_{p} \times \beta_{q}\right)\left(\left[a_{1}, a_{2}\right] \times b\right) .
\end{aligned}
$$


Similarly, we have the equality for edges of the type $a \times\left[b_{1}, b_{2}\right]$. Here $\beta_{p}$ and $\beta_{q}$ denote the canonical cochains that define the Berstein-Schwarz classes of $\mathbb{Z}_{p}$ and $\mathbb{Z}_{q}$. Thus, $\delta^{\prime}([\theta])=\left(\beta_{p} \times l, k \times \beta_{q}\right)$ in

$$
\begin{aligned}
H^{1}\left(\mathbb{Z}_{p q}, I(p) \otimes \mathbb{Z}(q)\right) \oplus H^{1}\left(\mathbb{Z}_{p q}, \mathbb{Z}(p) \otimes I(q)\right) & \\
& =H^{1}\left(\mathbb{Z}_{p q}, I(p) \otimes \mathbb{Z}(q) \oplus \mathbb{Z}(p) \otimes I(q)\right) .
\end{aligned}
$$

\subsection{Proof of Theorem 5.1}

We show that $u^{*}\left(\beta_{p q}^{n+1}\right)=0$, where

$$
u=i_{p} \times i_{q}: L_{p}^{n} \times L_{q}^{n} \rightarrow B \mathbb{Z}_{p} \times B \mathbb{Z}_{q}=B \mathbb{Z}_{p q}
$$

is the inclusion. Note that $\left(\beta_{p} \times l t_{q}, k t_{p} \times \beta_{q}\right)=\beta_{p} \times l t_{q}+k t_{p} \times \beta_{q}$. Thus, it suffices to show that $u^{*}\left(\beta_{p} \times l t_{q}+k t_{p} \times \beta_{q}\right)^{n+1}=0$. Note that

$$
u^{*}\left(\beta_{p} \times l+k \times \beta_{q}\right)=i_{p}^{*}\left(\beta_{p}\right) \times l+k \times i_{q}^{*}\left(\beta_{q}\right) .
$$

Then $\left(i_{p}^{*}\left(\beta_{p}\right) \times l+k \times i_{q}^{*}\left(\beta_{q}\right)\right)^{n+1}=\left(i_{p}^{*}\left(\beta_{p}\right) \times l\right)^{n+1}+\left(k \times i_{q}^{*}\left(\beta_{q}\right)\right)^{n+1}+F$, where $F$ consists of monomials containing both factors.

Claim $1\left(i_{p}^{*}\left(\beta_{p}\right) \times l\right)^{n+1}=0$ and $\left(k \times i_{q}^{*}\left(\beta_{q}\right)\right)^{n+1}=0$.

Proof There is an automorphism of the coefficients

$$
(I(p) \otimes \mathbb{Z}(q)) \otimes \cdots \otimes(I(p) \otimes \mathbb{Z}(q)) \rightarrow I(p) \otimes \cdots \otimes I(p) \otimes \mathbb{Z}(q) \otimes \cdots \otimes \mathbb{Z}(q)
$$

that takes $\left(i_{p}^{*}\left(\beta_{p}\right) \times l\right)^{n+1}$ to $i_{p}^{*}\left(\beta_{p}\right)^{n+1} \times l^{n+1}=0$. Similarly, $\left(k \times i_{q}^{*}\left(\beta_{q}\right)\right)^{n+1}=0$.

Claim $2\left(i_{p}^{*}\left(\beta_{p}\right) \times l\right) A\left(k \times i_{q}^{*}\left(\beta_{q}\right)\right)=0$ for any $A$.

Proof Indeed, since $i_{p}^{*}\left(\beta_{p}\right)$ is divisible by $q$ (see Proposition 5.2),

$$
\left(i_{p}^{*}\left(\beta_{p}\right) \times l\right) A\left(k \times i_{q}^{*}\left(\beta_{q}\right)\right)=\left(\frac{1}{q}\left(i_{p}^{*}\left(\beta_{p}\right) \times l\right)\right) A q\left(k \times i_{q}^{*}\left(\beta_{q}\right)\right)=0 .
$$

Thus, $F=0$ and the result follows.

\section{On the category of $\mathrm{ko}$-inessential manifolds}

\subsection{Deformation into the (n-2)-dimensional skeleton}

We recall that a classifying map $u: M \rightarrow B \pi$ of a closed orientable $n$-manifold $M$ can be deformed into the $(n-1)$-skeleton $B \pi^{(n-1)}$ if and only if $u_{*}([M])=0$, where $[M] \in H_{n}(M ; \mathbb{Z})$ denotes an integral fundamental class; see Babenko [1]. In [3] we 
proved the following proposition, which sets the stage for computation of the second obstruction.

Proposition 6.1 Every inessential $n$-manifold $M$ with a fixed $C W$ structure admits a classifying map $u: M \rightarrow B \pi$ with $u(M) \subset B \pi^{(n-1)}$ and $u\left(M^{(n-1)}\right) \subset B \pi^{(n-2)}$.

We postpone the proof of the following lemma to the end of the section.

Lemma 6.2 For any group $\pi$ and $C W$ complex $B \pi$, for $n \geq 5$ the homomorphism induced by the quotient map

$$
p_{*}: \pi_{n}\left(B \pi, B \pi^{(n-2)}\right) \rightarrow \pi_{n}\left(B \pi / B \pi^{(n-2)}\right)
$$

factors through the group of coinvariants as $p_{*}=\bar{p}_{*} \circ q_{*}$,

$$
\pi_{n}\left(B \pi, B \pi^{(n-2)}\right) \stackrel{q_{*}}{\longrightarrow} \pi_{n}\left(B \pi, B \pi^{(n-2)}\right)_{\pi} \stackrel{\bar{p}_{*}}{\longrightarrow} \pi_{n}\left(B \pi / B \pi^{(n-2)}\right),
$$

where $\bar{p}_{*}$ is injective.

We recall that for a $\pi$-module $M$ the group of coinvariants is $M \otimes_{\mathbb{Z} \pi} \mathbb{Z}$.

Remark In the proof of [3, Lemma 4.1] it was stated erroneously that $\bar{p}_{*}$ is bijective. It turns out that the injectivity of $\bar{p}_{*}$ was sufficient for the proof of that lemma to be carried out. Thus, due to Lemma 6.2 the results of [3] that depend on the lemma remain intact.

Theorem 6.3 Let $M$ be an $n$-manifold with a $C W$ complex structure with one topdimensional cell. Suppose that a classifying map $u: M \rightarrow B \pi$ satisfies the condition $u\left(M^{(n-1)}\right) \subset B \pi^{(n-2)}$ and let $\bar{u}: M / M^{(n-1)}=S^{n} \rightarrow B \pi / B \pi^{(n-2)}$ be the induced map. Then the following are equivalent:

(1) There is a deformation of $u$ in $B \pi$ to a map $f: M \rightarrow B \pi^{(n-2)}$.

(2) $\bar{u}_{*}(1)=0$ in $\pi_{n}\left(B \pi / B \pi^{(n-2)}\right)$, where $1 \in \mathbb{Z}=\pi_{n}\left(S^{n}\right)$.

Proof The primary obstruction to deforming $u$ to $B \pi^{(n-2)}$ is defined by the cocycle

$$
c_{u}=u_{*}: \pi_{n}\left(M, M^{(n-1)}\right) \rightarrow \pi_{n}\left(B \pi, B \pi^{(n-2)}\right)
$$

with the cohomology class $o_{u}=\left[c_{u}\right] \in H^{n}\left(M ; \pi_{n}\left(B \pi, B \pi^{(n-2)}\right)\right)$. By Poincaré duality, $o_{u}$ is dual to the homology class $P D\left(o_{u}\right) \in H_{0}\left(M ; \pi_{n}\left(B \pi, B \pi^{(n-2)}\right)\right)=$ $\pi_{n}\left(B \pi, B \pi^{(n-2)}\right)_{\pi}$ represented by $q_{*} u_{*}(1)$, where

$$
q_{*}: \pi_{n}\left(B \pi, B \pi^{(n-2)}\right) \rightarrow \pi_{n}\left(B \pi, B \pi^{(n-2)}\right)_{\pi}
$$


is the projection onto the group of coinvariants and

$$
u_{*}: \pi_{n}\left(M, M^{(n-1)}\right)=\mathbb{Z} \rightarrow \pi_{n}\left(B \pi, B \pi^{(n-2)}\right)
$$

is induced by $u$. We note that $\pi_{n}\left(B \pi, B \pi^{(n-2)}\right)=\pi_{n}\left(E \pi, E \pi^{(n-2)}\right)$. By Lemma 6.2 the homomorphism $\bar{p}_{*}$ is injective. Hence, $\bar{p}_{*} q_{*} u_{*}(1)=0$ if and only if $o_{u}=0$. The commutative diagram

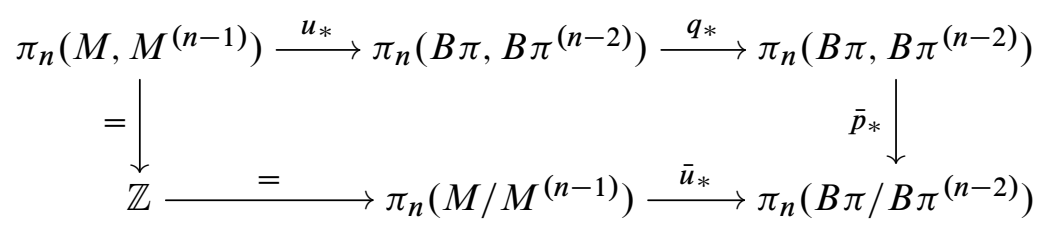

implies that $\bar{u}_{*}(1)=\bar{p}_{*} q_{*} u_{*}(1)$.

\section{2 ko-inessential manifolds}

We recall that an orientable, closed $n$-manifold $M$ is inessential if and only if $u_{*}([M])=0$, where $[M] \in H_{n}(M ; \mathbb{Z})$ is a fundamental class and $u: M \rightarrow B \pi$ is a classifying map. We call a closed spin $n$-manifold $M$ ko-inessential if $u_{*}\left([M]_{k o}\right)=0$ in $k o_{n}(B \pi)$, where $k o_{*}$ denotes the real connective $\mathrm{K}$-theory homology groups.

We recall that for every spectrum $E$ there is a natural morphism $S \rightarrow E$ of the spherical spectrum. This defines a natural transformation of corresponding (co)homology theories $\pi_{*}^{s} \rightarrow E_{*}$, where $\pi_{*}^{s}$ is the stable homotopy theory. In the case of $k o_{*}$ this natural transformation induces an isomorphism $\pi_{i}^{s}(\mathrm{pt}) \rightarrow k o_{i}(\mathrm{pt})$ for $i=0,1,2$. It allows us in some cases to reduce $k o_{*}$ problems to the stable homotopy groups.

We need the following proposition:

Proposition 6.4 [3] The natural transformation $\pi_{*}^{s}(\mathrm{pt}) \rightarrow k o_{*}(\mathrm{pt})$ induces an isomorphism $\pi_{n}^{s}\left(K / K^{(n-2)}\right) \rightarrow k o_{n}\left(K / K^{(n-2)}\right)$ for any $C W$ complex $K$.

We recall that spin manifolds are exactly those that admit an orientation in real connective $\mathrm{K}$-theory $k o_{*}$.

Theorem 6.5 A classifying map $u: M \rightarrow B \pi$ of an inessential, closed, spin $n-$ manifold $M, n>3$, is homotopic to a map $f: M \rightarrow B \pi^{(n-2)}$ if and only if $j_{*} u_{*}\left([M]_{k o}\right)=0$ in $k o_{n}\left(B \pi, B \pi^{(n-2)}\right)$, where $[M]_{k o}$ is a ko-fundamental class. 
Proof By Proposition 6.1 a classifying map $u$ can be chosen to satisfy the condition $u\left(M^{(n-1)}\right) \subset B \pi^{(n-2)}$. We show that $\bar{u}_{*}(1)=0$ if and only if $j_{*} u_{*}\left([M]_{k o}\right)=0$ and apply Theorem 6.3.

The restriction $n>3$ implies that $\bar{u}_{*}$ (1) survives in the stable homotopy group. In view of Proposition 6.4, the element $\bar{u}_{*}(1)$ survives in the composition

$$
\pi_{n}\left(B \pi / B \pi^{(n-2)}\right) \rightarrow \pi_{n}^{s}\left(B \pi / B \pi^{(n-2)}\right) \rightarrow k o_{n}\left(B \pi / B \pi^{(n-2)}\right) .
$$

The commutative diagram

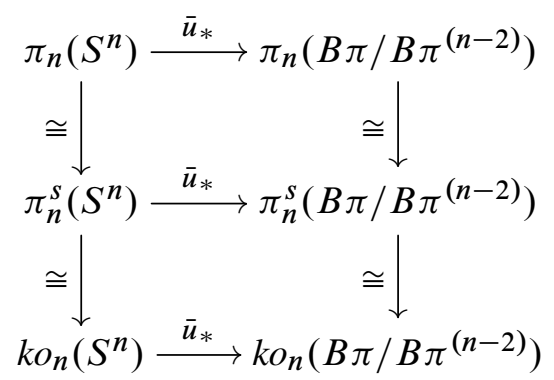

implies that $\bar{u}_{*}(1)=0$ for $k o_{n}$ if and only if $\bar{u}_{*}(1)=0$ for $\pi_{n}$.

From the diagram with the quotient map $\psi: M \rightarrow M / M^{(n-1)}=S^{n}$

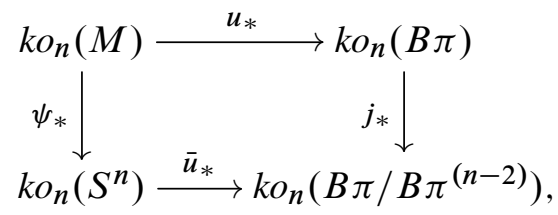

it follows that $j_{*} u_{*}\left([M]_{k o}\right)=\bar{u}_{*} \psi_{*}\left([M]_{k o}\right)=\bar{u}_{*}(1)$. Thus, $j_{*} u_{*}\left([M]_{k o}\right)=0$ if and only if $\bar{u}_{*}(1)=0$ for $n$-dimensional homotopy groups.

For spin manifolds we prove the following criterion:

Theorem 6.6 For a closed spin $n$-manifold $M$ with cat $M \leq \operatorname{dim} M-2$,

$$
j_{*} u_{*}\left([M]_{k o}\right)=0
$$

in $k o_{n}\left(B \pi, B \pi^{(n-2)}\right)$, where $u: M \rightarrow B \pi$ classifies the universal cover of $M$ and $j:(B \pi, \varnothing) \rightarrow\left(B \pi, B \pi^{(n-2)}\right)$ is the inclusion.

For a closed, spin, inessential $n$-manifold $M$ with $\pi_{2}(M)=0$, cat $M \leq \operatorname{dim} M-2$ if and only if $j_{*} u_{*}\left([M]_{k o}\right)=0$. 
Proof The inequality cat $M \leq n-2$ implies that the map $u$ has a lift $u^{\prime} \rightarrow G_{n-2}(B \pi)$ with $u=p_{n-2}^{\pi} u^{\prime}$. Since $G_{n-2}(B \pi)$ is homotopy equivalent to an (n-2)-dimensional complex, $p_{n-2}^{\pi}$ can be deformed to $p^{\prime}: G_{n-2}(B \pi) \rightarrow B \pi^{(n-2)}$. Thus $u$ can be deformed to $B \pi^{(n-2)}$. By Theorem $6.5, j_{*} u_{*}\left([M]_{k o}\right) \neq 0$.

Now let $\pi_{2}(M)=0$ and $j_{*} u_{*}\left([M]_{k o}\right)=0$. By Theorem 6.5 the map $u$ can be deformed to a map $f: M \rightarrow B \pi^{(n-2)}$. By Proposition 4.3, $\operatorname{cat}(u) \leq n-2$. Since $\pi_{2}(M)=0$, the universal covering of $M$ is 2 -connected. By Theorem 4.4, cat $M \leq n-2$.

Proposition 6.7 Let $M=L_{p}^{m} \times L_{q}^{n}, m, n>2$, be given a ko-orientation for some relatively prime $p$ and $q$ and let $u: M \rightarrow B \mathbb{Z}_{p q}$ be a classifying map of its universal cover. Then $u_{*}\left([M]_{k o}\right)=0$.

Proof Note that $[M]_{k o}= \pm(1+v)\left(\left[L_{p}^{m}\right]_{k o} \times\left[L_{q}^{n}\right]_{k o}\right)$, where $v \in \tilde{k_{0}}{ }^{0}(M)$ is in the reduced $k o$-theory and the product is the cap product (see [18, Chapter 5, Proposition 1.9]). Therefore it suffices to show that $u_{*}^{p}\left(\left[L_{p}^{m}\right]_{k o}\right) \times u_{*}^{q}\left(\left[L_{q}^{n}\right]_{k o}\right)=0$, where $u^{p}: L_{p}^{m} \rightarrow B \mathbb{Z}_{p}$ and $u^{q}: L_{q}^{n} \rightarrow B \mathbb{Z}_{q}$ are classifying maps. This equality follows from the fact that $k o_{m}\left(B \mathbb{Z}_{p}\right)$ is $q$-divisible and $k o_{n}\left(B \mathbb{Z}_{q}\right)$ is a $q$-torsion group.

Corollary 6.8 For $m, n>2$ and odd, relatively prime $p$ and $q$, or for $p$ odd and $q$ even with $n=2 k-1$ for even $k$, we have

$$
\operatorname{cat}\left(L_{p}^{m} \times L_{q}^{n}\right) \leq m+n-2 .
$$

Proof In this case the lens spaces are spin [11] and we can apply Proposition 6.7. Then Theorem 6.6 and the fact that $\pi_{2}\left(L_{p}^{m} \times L_{q}^{n}\right)=0$ imply the result.

For $m=n=3$ we obtain a different proof of Corollary 4.2:

Corollary 6.9 $\operatorname{cat}\left(L_{p}^{3} \times L_{q}^{3}\right)=4$ for all relatively prime $p$ and $q$.

\subsection{Coinvariants}

The following lemma can be found in [6, Lemma 3.3]:

Lemma 6.10 A commutative diagram with exact rows

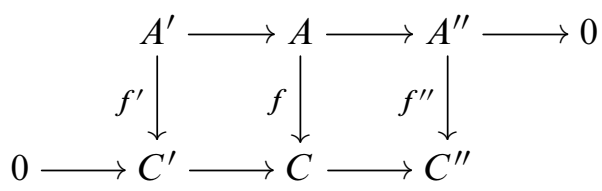

defines an exact sequence

$$
\operatorname{ker}\left(f^{\prime}\right) \rightarrow \operatorname{ker}(f) \rightarrow \operatorname{ker}\left(f^{\prime \prime}\right) \rightarrow \operatorname{coker}\left(f^{\prime}\right) \rightarrow \operatorname{coker}(f) \rightarrow \operatorname{coker}\left(f^{\prime \prime}\right) .
$$


Let $p: E \pi \rightarrow B \pi$ be the universal covering. Thus $p$ is the projection onto the orbit space of a free cellular $\pi$-action. Below we use the following abbreviations: $\pi=\pi_{1}(B), B=B \pi, B^{k}=B^{(k)}, E=E \pi$ and $E^{k}=E \pi^{(k)}$.

Proposition 6.11 $p_{*}: \pi_{n}\left(E / E^{n-1}\right) \rightarrow \pi_{n}\left(B / B^{n-1}\right)$ is an epimorphism.

Proof In the commutative diagram

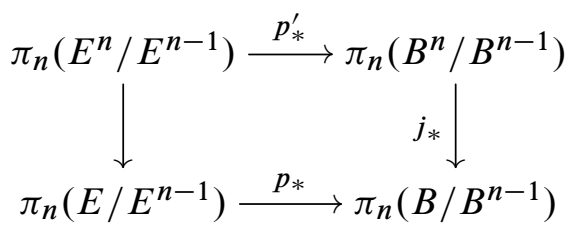

the homomorphisms $p_{*}^{\prime}$ and $j_{*}$ are epimorphisms. The former is surjective since it is induced by a retraction of a wedge of an $n$-sphere onto a smaller wedge; the latter is surjective due to the cellular approximation theorem. Therefore, $p_{*}$ is an epimorphism.

Recall that $\pi_{*}^{s}$ denotes the stable homotopy groups.

Corollary 6.12 For $n \geq 5$, the induced homomorphism

$$
p_{*}^{\prime}: \pi_{n}^{s}\left(E, E^{n-1}\right) \rightarrow \pi_{n}^{s}\left(B, B^{n-1}\right)
$$

is an epimorphism.

Proof This follows from the obvious natural isomorphisms

$$
\begin{aligned}
& \pi_{n}\left(E / E^{n-1}\right)=\pi_{n}^{s}\left(E / E^{n-1}\right)=\pi_{n}\left(E, E^{n-1}\right), \\
& \pi_{n}\left(B / B^{n-1}\right)=\pi_{n}^{s}\left(B / B^{n-1}\right)=\pi_{n}\left(B, B^{n-1}\right) .
\end{aligned}
$$

\subsection{Proof of Lemma 6.2}

For $n \geq 5$, the induced homomorphism

$$
p_{*}: \pi_{n}\left(B, B^{n-2}\right) \rightarrow \pi_{n}\left(B / B^{n-2}\right)
$$

factors through the group of coinvariants as $p_{*}=\bar{p}_{*} \circ q_{*}$,

$$
\pi_{n}\left(B, B^{n-2}\right) \stackrel{q_{*}}{\longrightarrow} \pi_{n}\left(B, B^{n-2}\right)_{\pi} \stackrel{\bar{p}_{*}}{\longrightarrow} \pi_{n}\left(B / B^{n-2}\right),
$$

where $\bar{p}_{*}$ is injective. 
Note that, for $n \geq 5$,

$$
\pi_{n}\left(B, B^{n-2}\right)=\pi_{n}\left(E, E^{n-2}\right)=\pi_{n}^{s}\left(E, E^{n-2}\right), \quad \pi_{n}\left(B / B^{n-2}\right)=\pi_{n}^{s}\left(B, B^{n-2}\right),
$$

and the composition

$$
\pi_{n}\left(B, B^{n-2}\right) \stackrel{q_{*}}{\longrightarrow} \pi_{n}\left(B, B^{n-2}\right)_{\pi} \stackrel{\bar{p}_{*}}{\longrightarrow} \pi_{n}\left(B / B^{n-2}\right)
$$

coincides with

$$
\pi_{n}^{s}\left(E, E^{n-2}\right) \stackrel{q_{*}}{\longrightarrow} \pi_{n}^{s}\left(E, E^{n-2}\right)_{\pi} \stackrel{\bar{p}_{*}}{\longrightarrow} \pi_{n}^{s}\left(B, B^{n-2}\right),
$$

where

$$
\bar{p}_{*} \circ q_{*}=p_{*}: \pi_{n}^{s}\left(E, E^{n-2}\right) \rightarrow \pi_{n}^{s}\left(B, B^{n-2}\right)
$$

is the homomorphism induced by the projection $p$.

Also note that $\pi_{*}^{s}\left(E, E^{i}\right)$ inherits a $\pi$-module structure via the $\pi$-action.

We extract from the diagram generated by $p$ and exact $\pi_{*}^{S}$-homology sequence of the triple $\left(E^{n}, E^{n-1}, E^{n-2}\right)$ the following two diagrams:

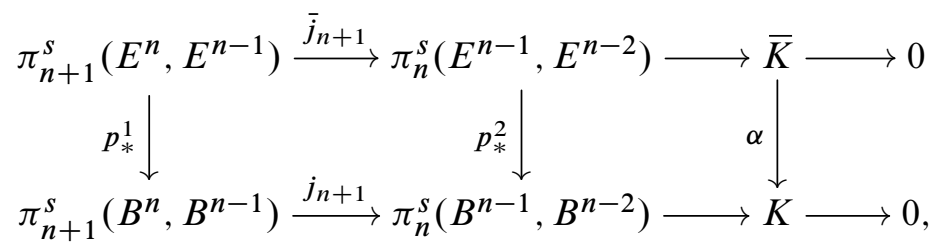

where $K$ and $\bar{K}$ are the cokernels of $j_{n+1}$ and $\bar{j}_{n+1}$, and

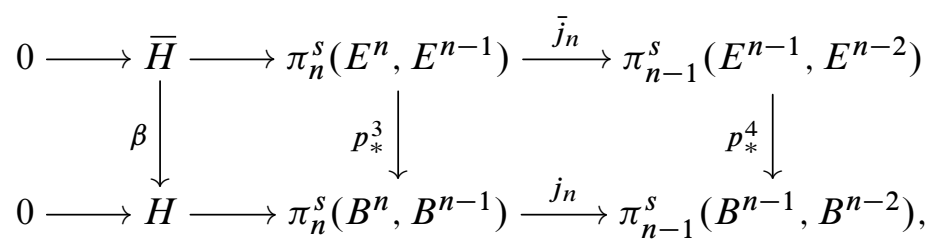

where $H$ and $\bar{H}$ are the kernels of $j_{n}$ and $\bar{j}_{n}$. Note that the homomorphisms $p_{*}^{3}$ and $p_{*}^{4}$ are the direct sums of the augmentation homomorphism

$$
\epsilon: \mathbb{Z} \pi \rightarrow \mathbb{Z}
$$

The homomorphisms $p_{*}^{1}$ and $p_{*}^{2}$ are direct sums of the mod 2 augmentation homomorphisms

$$
\bar{\epsilon}: \mathbb{Z}_{2} \pi \rightarrow \mathbb{Z}_{2}
$$


Also note that $p_{*}^{i} \otimes_{\pi} 1_{\mathbb{Z}}$ is an isomorphism for $i=1,2,3,4$. Taking the tensor product of the first diagram with $\mathbb{Z}$ over $\mathbb{Z} \pi$ would give a commutative diagram with the two left vertical arrows isomorphisms. Then, by the five lemma, $\alpha^{\prime}=\alpha \otimes_{\pi} 1_{\mathbb{Z}}$ is an isomorphism.

We argue that $\beta^{\prime}=\beta \otimes_{\pi} 1_{\mathbb{Z}}$ is a monomorphism. Note that $\operatorname{ker}(\beta) \subset \operatorname{ker}\left(p_{*}^{3}\right)=\bigoplus I(\pi)$, where $I(\pi)$ is the augmentation ideal.

Claim

$$
\operatorname{ker}(\beta) \otimes_{\pi} \mathbb{Z}=0 .
$$

Proof We show that $x \otimes_{\pi} 1=0$ for all $x \in \operatorname{ker}(\beta)$. Let $x=\sum x_{i}, x_{i} \in I(\pi)$. It suffices to show that $x_{i} \otimes_{\pi} 1=0$ for all $x_{i}$. Note that $x_{i}=\sum n_{j}\left(\gamma_{j}-e\right), \gamma_{j} \in \pi$, $n_{J} \in \mathbb{Z}$. Note that $(\gamma-e) \otimes_{\pi} 1=0$ since

$$
(\gamma-e) \otimes_{\pi} 1=\gamma \otimes_{\pi} 1-e \otimes_{\pi} 1=\gamma\left(e \otimes_{\pi} 1\right)-e \otimes_{\pi} 1=e \otimes_{\pi} \gamma(1)-e \otimes_{\pi} 1=0 .
$$

The tensor product with $\mathbb{Z}$ over $\mathbb{Z} \pi$ of the exact sequence

$$
\operatorname{ker}(\beta) \rightarrow \bar{H} \rightarrow \operatorname{im}(\beta) \rightarrow 0
$$

implies that

$$
\beta_{0}=\beta \otimes \mathrm{id}: \bar{H} \otimes_{\pi} \mathbb{Z}=\bar{H}_{\pi} \rightarrow \operatorname{im}(\beta) \otimes_{\pi} \mathbb{Z}=\operatorname{im}(\beta)
$$

is an isomorphism. The latter equality follows from the fact that both $\operatorname{im}(\beta)$ and $\mathbb{Z}$ are trivial $\pi$-modules. Then $\beta^{\prime}$ is a monomorphism as the composition of an isomorphism $\beta_{0}$ and the inclusion $\operatorname{im}(\beta) \rightarrow H$.

We consider the diagram of short exact sequences:

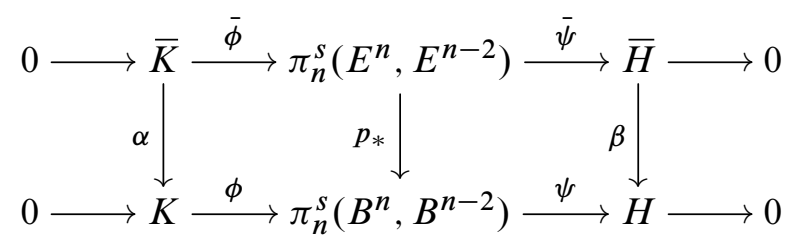

Then we apply the tensor product with $\mathbb{Z}$ over $\mathbb{Z} \pi$ to this diagram to obtain the following commutative diagram with exact rows:

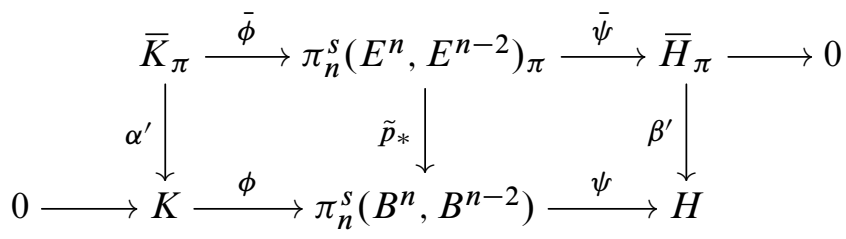

Lemma 6.10 implies that $\tilde{p}_{*}$ is a monomorphism. 
Next we consider the diagram generated by $\left(E, E^{n}, E^{n-2}\right)$ and $\left(B, B^{n}, B^{n-2}\right)$,

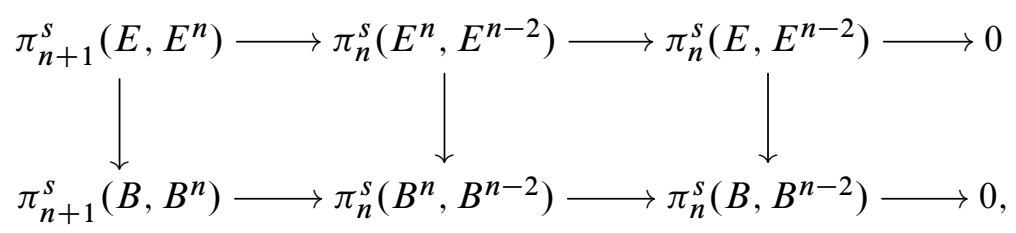

and tensor it with $\mathbb{Z}$ over $\mathbb{Z} \pi$ to obtain the following commutative diagram with exact rows:

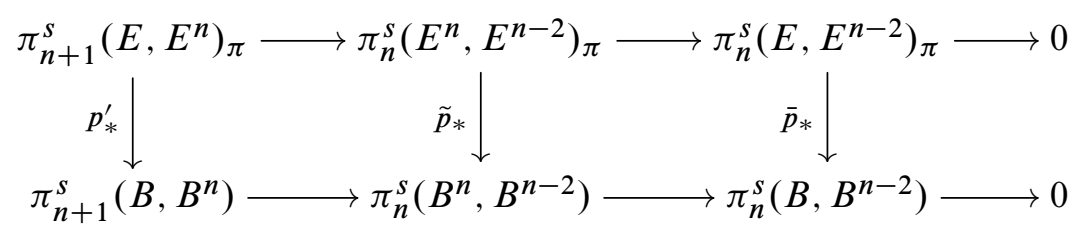

Since $p_{*}^{\prime}$ is an epimorphism (see Corollary 6.12) and $\tilde{p}_{*}$ is a monomorphism by the monomorphism version of the five lemma, we obtain that $\bar{p}_{*}$ is a monomorphism.

\section{On the category of the sum}

The following theorem was proven by R Newton [16] under the assumption that cat $M$, cat $N>2$.

Theorem 7.1 For closed manifolds $M$ and $N$ there is the inequality

$$
\operatorname{cat}(M \# N) \leq \max \{\text { cat } M, \text { cat } N\} .
$$

His proof is based on obstruction theory. Here we present a proof that works in full generality. Our proof is an application of the following:

Theorem 7.2 (W Singhof [21, Theorem 4.4]) For any closed $n$-manifold $M$ with cat $M=k \geq 2$, there is a categorical partition $Q_{0}, \ldots, Q_{k}$ into manifolds with boundary such that $Q_{i} \cap Q_{j}$ is an (n-1)-manifold with boundary (possibly empty) for all $i, j$ and each $Q_{i}$ admits a deformation retraction onto an $(n-k)$-dimensional CW complex.

For $B \subset A \subset X$, a homotopy $H: A \times I \rightarrow X$ is called a deformation of $A$ in $X$ onto $B$ if $H_{A \times\{0\}}=1_{A}, H(A \times\{1\})=B$, and $H(b, t)=b$ for all $b \in B$ and $t \in I=[0,1]$. The following is well known: 
Proposition 7.3 Let $A \subset M$ be a subset contractible to a point in an $m$-manifold $M$ and let $B \subset A$ be a closed $n-b a l l$ which admits a regular neighborhood. Then there is a deformation of $A$ in $M$ onto $B$.

Proof of Theorem 7.1 Let $n=\operatorname{dim} M=\operatorname{dim} N$. Suppose that cat $M$, cat $N \leq k$. We show that $\operatorname{cat}(M \# N) \leq k$. If $k=1$, the statement obviously follows from the fact that $M$ and $N$ are homeomorphic to the sphere. We assume that $k \geq 2$. Let $Q_{0}, \ldots Q_{k}$ be a partition of $M$ into $M$-contractible subsets as in Singhof's theorem. We may assume that $Q_{0} \cap Q_{1} \neq \varnothing$. Moreover, we may assume that there is a closed topological $n$-ball $D \subset Q_{0} \cup Q_{1}$ with a collar in $Q_{0} \cup Q_{1}$ and $D_{0}=D \cap Q_{0}, D_{1}=D \cap Q_{1}$ such that the triad $\left(D, D_{0}, D_{1}\right)$ is homeomorphic to the triad $\left(B, B_{+}, B_{-}\right)$, where $B$ is the unit ball in $\mathbb{R}^{n}, B_{+}=B \cap \mathbb{R}_{+}^{n}, B_{-}=B \cap \mathbb{R}_{-}^{n}$, and $\mathbb{R}_{+}^{n}=\left\{\left(x_{1}, \ldots x_{n}\right) \in \mathbb{R}^{n} \mid x_{n} \geq 0\right\}$ and $\mathbb{R}_{-}^{n}=\left\{\left(x_{1}, \ldots x_{n}\right) \in \mathbb{R}^{n} \mid x_{n} \leq 0\right\}$ are the half-spaces. Additionally we may assume that the collar of $D$ intersected with $Q_{0} \cap Q_{1}$ defines a collar of $D \cap Q_{0} \cap Q_{1}$ in $Q_{0} \cap Q_{1}$.

Similarly, we may assume that there is a categorical partition $V_{0}, \ldots, V_{k}$ of $N$ as in Theorem 7.2 and a closed $n$-ball $D^{\prime}$ with a collar such that the triad $\left(D^{\prime}, D_{0}^{\prime}, D_{1}^{\prime}\right)$ is homeomorphic to the triad $\left(B, B_{+}, B_{-}\right)$, where $D_{0}^{\prime}=D^{\prime} \cap V_{0}, D_{1}^{\prime}=D^{\prime} \cap V_{1}$.

We may assume that the connected sum $M \# N$ is realized as a subset $M \# N=$ $M \cup N \backslash$ Int $D \subset M \cup_{h} N$ for some homeomorphism $h: D^{\prime} \rightarrow D$ that preserves the triad structures.

Let $W_{0}=\left(Q_{0} \backslash \operatorname{Int} D\right) \cup\left(V_{0} \backslash\right.$ Int $\left.D^{\prime}\right), W_{1}=\left(Q_{1} \backslash \operatorname{Int} D\right) \cup\left(V_{1} \backslash\right.$ Int $\left.D^{\prime}\right)$ and $W_{i}=Q_{i} \cup V_{i}$ for $i=2, \ldots, k$. Note that $Q_{i} \cap V_{i}=\varnothing$ for $i \geq 2$. By Singhof's theorem each $Q_{i}$ can be deformed to an $(n-k)$-dimensional subset $S_{i}$ contractible in $M$. Since $k \geq 2$, there is a contraction of $S_{i}$ to a point in $M$ that misses a given point. Hence, there is a contraction of $S_{i}$ to a point in $M$ that misses the ball $D$. Thus $Q_{i}$ for $i \geq 2$ can be contracted to a point in $M \# N$. Similarly, for $i \geq 2$ the set $V_{i}$ can be contracted to a point in $M \# N$. Hence the sets $W_{i}$ for $i \geq 2$ are categorical.

Let $A_{i}=Q_{i} \cap \partial D$ for $i=0,1$. We show that there is a deformation of $Q_{i} \backslash$ Int $D$ in $M \# N$ to $A_{i}$. The collar of $Q_{i} \cap D$ in $Q_{i}$ allows us to construct a homeomorphism of $Q_{i} \backslash$ Int $D$ to $Q_{i}$ homotopic to the identity. Hence $Q_{i} \backslash$ Int $D$ can be deformed onto an $(n-k)$-dimensional subset $S_{i}$ contractible in $M$. A contraction of $S_{i}$ to a point can be chosen missing $c_{0} \in \operatorname{Int} D$. By Proposition 7.3 there is a deformation of $Q_{i} \backslash \operatorname{Int} D$ in $M \backslash\left\{c_{0}\right\}$ onto $A_{i}$ fixing $A_{i}$. Similarly, for $i=0,1$ there is a deformation of $V_{i} \backslash$ Int $D^{\prime}$ in $N \backslash\left\{c_{0}^{\prime}\right\}$ to $A_{i}=V_{i} \cap \partial D^{\prime}$ fixing $A_{i}$ where $c_{0}^{\prime} \in \operatorname{Int} D^{\prime}$. Applying the radial projections from $c_{0}$ and $c_{0}^{\prime}$ gives us such deformations in $M \# N$. Pasting these two deformations defines a deformation of $W_{i}, i=0,1$, in $M \# N$ to $A_{i}$. Since the sets $A_{i}$ are contractible, it follows that the sets $W_{i}, i=0,1$. are categorical. 


\section{References}

[1] I K Babenko, Asymptotic invariants of smooth manifolds, Izv. Ross. Akad. Nauk Ser. Mat. 56 (1992) 707-751 MR1208148 In Russian; translated in Russian Acad. Sci. Izv. Math. 41 (1993) 1-38

[2] I Berstein, On the Lusternik-Schnirelmann category of Grassmannians, Math. Proc. Cambridge Philos. Soc. 79 (1976) 129-134 MR0400212

[3] D Bolotov, A Dranishnikov, On Gromov's scalar curvature conjecture, Proc. Amer. Math. Soc. 138 (2010) 1517-1524 MR2578547

[4] G E Bredon, Sheaf theory, 2nd edition, Graduate Texts in Mathematics 170, Springer, New York (1997) MR1481706

[5] K S Brown, Cohomology of groups, Graduate Texts in Mathematics 87, Springer, New York (1994) MR1324339

[6] H Cartan, S Eilenberg, Homological algebra, Princeton Univ. Press, Princeton, NJ (1956) MR0077480

[7] O Cornea, G Lupton, J Oprea, D Tanré, Lusternik-Schnirelmann category, Mathematical Surveys and Monographs 103, Amer. Math. Soc. (2003) MR1990857

[8] A N Dranishnikov, M Katz, Y B Rudyak, Small values of the Lusternik-Schnirelman category for manifolds, Geom. Topol. 12 (2008) 1711-1727 MR2421138

[9] A N Dranishnikov, Y B Rudyak, On the Berstein-Svarc theorem in dimension 2, Math. Proc. Cambridge Philos. Soc. 146 (2009) 407-413 MR2475974

[10] J Ewing, S Moolgavkar, L Smith, R E Stong, Stable parallelizability of lens spaces, J. Pure Appl. Algebra 10 (1977/78) 177-191 MR0478152

[11] A Franc, Spin structures and Killing spinors on lens spaces, J. Geom. Phys. 4 (1987) 277-287 MR957015

[12] M Gromov, Filling Riemannian manifolds, J. Differential Geom. 18 (1983) 1-147 MR697984

[13] M Katz, Y B Rudyak, Lusternik-Schnirelmann category and systolic category of lowdimensional manifolds, Comm. Pure Appl. Math. 59 (2006) 1433-1456 MR2248895

[14] A A Kosinski, Differential manifolds, Pure and Applied Mathematics 138, Academic Press, Boston (1993) MR1190010

[15] J H Kwak, The stable parallelizability of a smooth homotopy lens space, J. Pure Appl. Algebra 50 (1988) 155-169 MR938019

[16] R Newton, On Lusternik-Schnirelmann category of connected sums, preprint arXiv: 1205.0216

[17] P Olum, Mappings of manifolds and the notion of degree, Ann. of Math. 58 (1953) 458-480 MR0058212 
[18] Y B Rudyak, On Thom spectra, orientability, and cobordism, Springer, Berlin (1998) MR1627486

[19] Y B Rudyak, On category weight and its applications, Topology 38 (1999) 37-55 MR1644063

[20] J-P Serre, Homologie singulière des espaces fibrés: applications, Ann. of Math. 54 (1951) 425-505 MR0045386

[21] W Singhof, Minimal coverings of manifolds with balls, Manuscripta Math. 29 (1979) 385-415 MR545050

[22] A S Švarc, The genus of a fibered space, Trudy Moskov. Mat. Obšč. 10, 11 (1961, 1962) 217-272, 99-126 MR0154284 In Russian; translated in Amer. Math. Soc. Transl. 55 (1966) 49-140

[23] C T C Wall, Surgery on compact manifolds, 2nd edition, Mathematical Surveys and Monographs 69, Amer. Math. Soc. (1999) MR1687388

Department of Mathematics, University of Florida 358 Little Hall, Gainesville, FL 32611-8105, USA

dranish@math.ufl.edu

Received: 15 October 2014 Revised: 17 February 2015 